\title{
Simulating Polymer Mass Transfer by Analogy to Heat Transfer at Very Large Prandtl Numbers
}

\author{
J. J. White, T. L. Jeans, A. G. L. Holloway \\ Department of Mechanical Engineering, University of New Brunswick, Fredericton, Canada \\ White.JJ@unb.ca
}

\begin{abstract}
It is known that a small concentration of polymer in the boundary layer can produce significant drag reduction. This has been studied extensively for internal flow and polymer injection in an external flow; though much is still not understood. Significant effort has focused on the PEG poly(ethylene glycol) / PEO poly(ethylene oxide) polymer for drag reduction. More recently external flow research has focused on drag reduction for ships. This work focuses on the novel use of an ablative polymer paint to induce drag reduction on submarine geometries. In order to simulate polymer drag reduction from an ablative polymer, first the polymer mass flux from the surface must be determined and then the polymer must be tracked as it is convected along the surface of the hull. To do this, polymer concentration is treated as a passive scalar. However, there are very few experimental high Schmidt number studies of mass transfer available and most computational fluid dynamics solvers do not have well developed passive scalar transport models. Alternatively, one can use the heat transfer models and the analogy between heat and mass transfer. It therefore becomes necessary to quantify the equivalencies between polymer concentration and temperature. It is known that approximate concentrations of PEG from $10-200 \mathrm{wppm}$ at the wall can generate drag reduction with $200 \mathrm{wppm}$ reaching the maximum drag reduction asymptote. In the analogy, this equates to a temperature rise $\Delta \mathrm{T}$ of $2 \cdot 4 \cdot 10^{-6}$ to $4.8 \cdot 10^{-5}{ }^{\circ} \mathrm{K}$ in water, a very small equivalent temperature rise is able to cause a significant drag reduction in the analogy. High molecular weight polymers are typically used in drag reduction applications, for example being Dow Chemical WSR301 a PEG polymer of molecular weight of $4 \cdot 10^{6} \mathrm{~g} / \mathrm{mol}$. Polymer theory can predict, for a random coil polymer in a solvent, a diffusivity from the Stokes-Einstein equation. An estimate for diffusivity for very low concentrations of PEG for molecular weight $4 \cdot 10^{6}$ in water will be on the order of $10^{-12} \mathrm{~m}^{2} / \mathrm{s}$. This results in an equivalent Prandtl number $(\operatorname{Pr})$ on the order of $10^{6}$. Mass transfer of benzoic acid dissolving into glycerine-water solutions is known to approach an analogous $\operatorname{Pr}$ of $10^{6}$. $\operatorname{Pr}>>10^{2}$ have not been studied extensively, as oils are only in the 100s. The finite volume solver has been verified against flat plate heat transfer for air, water, and technical oil. Results will be presented for Pr up to $10^{6}$ under steady flow.
\end{abstract}

